\title{
Intrinsic QRS Duration
}

National Cancer Institute

\section{Source}

National Cancer Institute. Intrinsic QRS Duration. NCI Thesaurus. Code C100060.

The duration of the QRS complex that is generated by the subject's own heart. (ACC) 